\title{
Use of single-locus DNA probes in the establishment of relatedness in wild populations
}

\author{
J. F. Y BROOKFIELD* \& D. T. PARKIN \\ Department of Genetics, University of Nottingham, Queens Medical Centre, Nottingham NG7 2UH, U.K.
}

\begin{abstract}
We examined the use of hypervariable single-locus DNA probes in providing evidence of the form of relatedness of pairs of individuals from wild populations. We asked how many loci are required in order to have a good chance of finding evidence that would significantly distinguish between alternative relationship hypotheses. The number of loci required is unrealistically large if we wish to find significant evidence to resolve relationships of greater than the first degree.
\end{abstract}

Keywords: DNA profiling, likelihood ratio.

\section{Introduction}

It has become clear that the genomes of a large range of eukaryotic organisms contain loci consisting of short, tandem repeats which display high variability in copy number (Jeffreys et al., 1985a,b). These have been called minisatellite or VNTR loci. These have proved very useful in the human context for the establishment of paternity and of identity in criminal cases (Gill et al., 1985; Jeffreys et al., 1985c). Correspondingly, they can be used to demonstrate paternity in a variety of animal species (Wetton et al., 1987; Gyllensten et al., 1989). While these sequences are very powerful in the establishment of first-degree relatedness (Brookfield, 1989), they can be shown (Lynch, 1988; Brookfield, 1991) to be considerably less powerful and, indeed, almost useless, in the establishment of more distant degrees of relatedness. However, for a variety of applications it is hoped that probes visualizing VNTR sequences individually (single-locus probes) will be able to distinguish more distant degrees of relatedness as, by raising indefinitely the number of single-locus probes utilized, the technique would appear to be capable of being made arbitrarily powerful. Here we assess the number of minisatellite loci required to have a good probability of distinguishing between various relationship hypotheses. We find that an unrealistically large number of loci would be required before it would be possible to distinguish with confidence between

*Correspondence. pairs of unrelated individuals and second- and thirddegree relatives.

\section{The model}

In this paper we will consider a situation in which two individuals are sampled from a population of sexuallyreproducing diploids. These individuals are then examined using a series of $n$ single-locus probes. At each of these $n$ loci, all the alleles have the same frequency of $q$, and none of the loci is sex-linked. Examination of the genotypes at each locus for the two individuals will provide evidence concerning their likely relationship. Potentially, they could be related in a number of ways: e.g. they could be cousins or siblings. From simple Mendelian inheritance, we can calculate the probability of an observed array of genotypes in the two individuals under any system of relatedness. For example, if we wish to decide whether our two individuals are cousins or siblings, we can first calculate the probability that they would have their observed genotypes if they were truly cousins, and then the probability if they were actually siblings. The ratio of these two probabilities (the likelihood ratio) shows the strength of the evidence provided by the genotypes to distinguish between the two alternative relationships. If this likelihood ratio is less than 0.05 , or greater than 20 , the data are considered to provide significant evidence to distinguish between the two alternatives. To turn the problem round, we can also assess the power of the technique to establish relatedness by asking how many 
loci we will need to have a better than 50 per cent chance of finding significant evidence in favour of one or other hypothesis.

Thus, if we have a pair of hypotheses $A$ and $B$ concerning the relationship between two individuals, and we have a data set $\mathrm{i}$, we calculate $P(\mathrm{i} \mid \mathrm{A})$ and $P(\mathrm{i} \mid \mathrm{B})$, the probabilities of getting the data set under the two hypotheses. The likelihood ratio for $\mathrm{i}$ $(\mathrm{LR}(\mathrm{i} \mid \mathrm{A}, \mathrm{B}))$ is thus $P(\mathrm{i} \mid \mathrm{A}) / P(\mathrm{i} \mid \mathrm{B})$. If hypothesis $\mathrm{A}$ is true, then the probability of getting this particular likelihood ratio is $P(\mathbf{i} \mid \mathrm{A})$. Thus, to assess the probability that we will get significant evidence in favour of hypothesis $\mathrm{A}$ compared with hypothesis $\mathrm{B}$ if $\mathrm{A}$ is correct, we sum the $P(\mathrm{i} \mid \mathrm{A})$ values for all $\mathrm{i}$ for which $\mathrm{LR}(\mathrm{i} \mid \mathrm{A}, \mathrm{B})$ is greater than 20 . We can describe the test as 'powerful' in resolving between these hypotheses if this probability of getting significant evidence in favour of the true hypothesis is greater than 50 per cent.

For a single-locus DNA probe the data set for two individuals can fall into one of seven different classes, which we can call classes 1 to 7 . Specifically, the possibilities are:

Class 1: both homozygous for the same allele.

Class 2: both homozygous but for different alleles.

Class 3: one homozygous, one heterozygous, with one allele shared.

Class 4: one homozygous, one heterozygous, with no shared allele.

Class 5: both heterozygous with both alleles shared.

Class 6: both heterozygous, but with only one allele shared.

Class 7: both heterozygous, but with no allele shared.

The probabilities of getting these classes depend on the type of relatedness of the two individuals. They can be calculated using the Cotterman $k$ coefficients (Cotterman, 1940) discussed in Crow \& Kimura (1970). Table 1 shows the probabilities for a range of eight types of relatedness. Note that the probabilities depend on the precise form of the relationship and not merely on the $r$ value (coefficient of relatedness), e.g. a parent-offspring pair has a different probability set from a pair of brothers.

The values given in Table 1 refer to a single locus. Over $n$ loci the probability of the particular combination of genotypes will be the product of the probabilities of the genotypes at the individual loci. As the number of loci increases, so does the number of genotype combinations. With $n$ loci there are $7^{n}$ combinations. Each of these combinations will have a probability that is the product of the probabilities at the individual loci and a likelihood ratio that is the product of the likelihood ratios at the individual loci. What we have done, therefore, for each pair of relationships, is to search by computer through all the $7^{\text {n }}$ combinations, and sum the probabilities of the genotype combinations which give likelihood ratios of over 20 . We increase the number of loci studied until the test becomes 'powerful' according to our above definition (when the summed probability increases above 50 per cent). The number of loci required obviously increases with the closeness of the relationship hypotheses being compared. The number also increases with the mean frequency of the alleles at the individual loci. Table 2 shows the numbers of loci required to supply a 'powerful' test with a $q$ values of 0.05 . We have only extended the analysis to nine loci because of the extensive computer time required for calculations that include larger numbers of loci. The compound nature of the statistic that we employ, namely that half the time the likelihood ratio is greater than 20 , causes some anomalies in the Table. There are cases in which a test deemed powerful with a smaller number of loci becomes non-powerful as the number of loci increases. While the mean likelihood ratio in favour of the true hypothesis increases as the number of loci increases, the increase in the proportion of time the likelihood ratio falls over 20 is not linear or even regular with increasing number of loci. Our concept of 'powerful' is a function of the probabilities of different genotypes and the likelihood ratio associated with them. Thus, for the case of truly unrelated individuals, the probability, with eight loci, that they have genotypes that give a likelihood ratio of 20 or more relative to the double first-cousins hypothesis is 52 per cent. The test is therefore defined as powerful. However, with nine loci the probability that there is a significant likelihood ratio drops to 47.3 per cent. Within these 47.3 per cent of cases, the mean likelihood ratio is, however, considerably increased relative to the situation with eight loci.

\section{Discussion}

Table 2 shows that quite a small number of single-locus probes is adequate to distinguish siblings or, especially, parent-offspring pairs. It also shows, however, that a very large number of loci, each of which has a low mean allelic frequency, is required before the technique can, for example, distinguish between cousins and unrelated individuals. Note that the test is highly asymmetrical, particularly in cases involving the parent-offspring relationship. The number of loci required to show that a parent and an offspring are not half-sibs is six, but the number of loci required to show half-sibs are not parent and offspring is two. This is because parents and offspring always, barring mutation, share at least one allele at each locus. If the true relationship is not parent-offspring then, even with a 

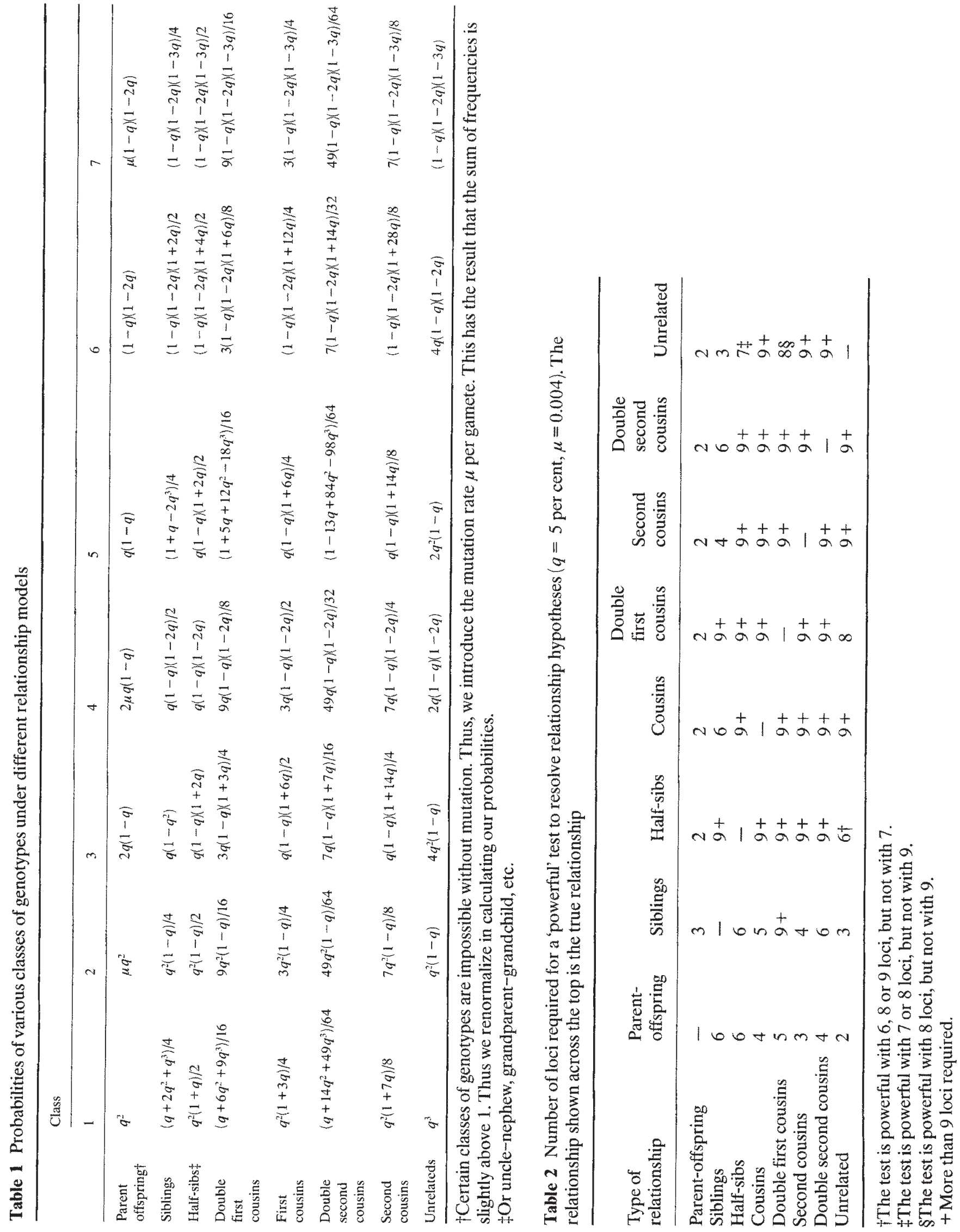
small number of loci, there is a high probability that no alleles are shared at one or more of the loci, and thus the likelihood given the parent-offspring hypothesis becomes a function of the mutation rate. This gives a significant likelihood ratio. In the reciprocal case, however, in which the relationship truly is parent-offspring, sharing of alleles given another hypothesis is a function of the frequencies of the alleles in the population, and thus a significant likelihood ratio is much less probable when the number of loci is small.

We have only considered situations in which up to nine loci are examined. The power of the technique can be extended by consideration of more and more loci and, eventually, one might imagine that all degrees of relatedness, however subtly different they may be, could be reliably distinguished. However, the ability of successive probes to add further information regarding relatedness relies on their being independent and thus genetically unlinked. Unfortunately, if we have 10 probes scattered over say, 20 chromosome pairs, the probability that all probes are on different chromosomes is only about 7 per cent.

We do not suggest that the single-locus probes which are described here are without use in the determination of relationships. The similarity between individuals in their minisatellite band patterns is very strongly correlated with genetic identity and, thus, when comparing large numbers of individuals, average band sharing will give a very clear identification of the average degree of relatedness.

Our analysis here is solely in terms of the likelihood ratio for pairs of alternative relationship hypotheses. These alternative hypotheses will usually have very different prior probabilities. The number of pairs of individuals who are cousins, for example, will normally be larger than the number of pairs who are siblings. The number of pairs that are unrelated would be very much larger still. If one were to produce a theoretical model of the population structure and make the assumption of random sampling from it, it would be possible to arrive at prior odds of different relationships and thus, by utilizing the likelihood ratios from the DNA profile evidence, calculate posterior odds for relationship alternatives. In practice, however, organisms to be examined using DNA profiling will have other evidence associated with them which will mean that they cannot be regarded as random samples from the population. Thus theoretical predictions of the prior odds, and the posterior odds derived from them, will never be accurate.

\section{References}

BROOKFIELD, J. F. Y. 1989. Analysis of DNA fingerprinting data in cases of disputed paternity. IMA J. Math. Appl. Med. Biol., 6, 111-131.

BROOKFIELD, J. F. Y. 1991. The statistical interpretation of hypervariable DNAs. In: Hewitt, G. M., Johnston, A. W. B. and Young, J. P. W., (eds), Molecular Techniques in Taxonomy. Springer-Verlag, Berlin, pp. 159-169.

COTTERMAN, c. w. 1940. A calculus for statistico-genetics. Unpublished thesis, Ohio State University, Columbus. $\mathrm{OH}$.

CROW, J. F. AND KIMURA, M. 1970. An Introduction to Population Genetics Theory. Harper and Row, New York, pp. 132-136.

GILL, P., JEFFREYS, A. J. AND WERRETT, D. J. 1985. Forensic application of DNA 'fingerprints'. Nature, 318, 577-579.

GYLLENSTEN, U. B., JAKOBSSON, S., TEMRIN, H. AND WILSON, A. C. 1989. Nucleotide sequence and genomic organisation of bird minisatellites. Nucl. Acids Res., 17, 2203-2214.

JEFFREYS, A. J., WILSON, V. AND THEIN, S. L. 1985a. Hypervariable 'minisatellite' regions in human DNA. Nature, 314, 67-73.

JEFFREYS, A. J., WILSON, v. AND THEIN, S. L. 1985b. Individualspecific 'fingerprints' in human DNA. Nature, 316, 76-79. JEFFREYS, A. J., BROOKFIELD, J. F. Y. AND SEMEONOFF, R. 1985c. Positive identification in an immigration test-case using human DNA fingerprints. Nature, 317, 818-819.

LYNCH, M. 1988. Estimation of relatedness by DNA fingerprinting. Mol. Biol. Evol., 5, 584-599.

WETTON, J. H., CARTER, R. E., PARKIN, D. T. AND WALTERS, D. 1987. Demographic study of a wild house sparrow population by DNA fingerprinting. Nature, 327, 147-149.

WONG, Z., WILSON, V., PATEL. I., POVEY, S. AND JEFFREYS, A. J. 1987. Characterization of a panel of highly variable minisatellites cloned from human DNA. Ann. Hum. Genet., 51, 259-288. 